\title{
Maturational decrease of glutamate in the human cerebral cortex from childhood to young adulthood: a ${ }^{1} \mathrm{H}-\mathrm{MR}$ spectroscopy study
}

\author{
Mami Shimizu ${ }^{1}$, Yuji Suzuki ${ }^{1}$, Kenichi Yamada ${ }^{1}$, Satoshi Ueki ${ }^{1}$, Masaki Watanabe ${ }^{1}$, Hironaka Igarashi ${ }^{1}$ and Tsutomu Nakada ${ }^{1}$
}

BACKGROUND: The aim of the present study was to investigate maturational changes in glutamate (Glu) in the human cerebral cortex from childhood to young adulthood using 3.0-Tesla proton magnetic resonance spectroscopy ('H-MRS), which is capable of quantifying Glu in vivo.

METHODS: Normal volunteers comprising 11 children (aged 4-13 years) and 11 young adults (aged 18-33 years) participated in the study. Single-voxel point-resolved spectroscopy (PRESS, repetition time/echo time $=2,000 / 80 \mathrm{~ms}$ ) was performed on the frontal and occipital cortices, and the Gluto-creatine ratio ( $\mathrm{Glu} / \mathrm{Cr}$ ) and $\mathrm{N}$-acetylaspartate-to-creatine ratio (NAA/Cr) were determined.

RESULTS: In both the frontal and occipital cortices, Glu/Cr was significantly lower during young adulthood relative to that during childhood. NAA/Cr did not differ significantly between the two age groups.

CONCLUSION: This study has provided objective evidence that cerebral cortical $\mathrm{Glu} / \mathrm{Cr}$ decreases between childhood and young adulthood. The observed decrease in $\mathrm{Glu} / \mathrm{Cr}$ may reflect the simultaneous occurrence of maturational changes, such as changes in cortical microstructure and the intercellular compartmentation of Glu metabolism.

$\mathbf{R}$ efinement of cortical networks has been proposed to have a pivotal role in postnatal maturation of the human brain until young adulthood. Dysregulation of this process is thought to be associated with a range of neurodevelopmental and psychiatric disorders. Therefore, to investigate and better understand the pathophysiology of these disorders, establishment of quantitative physiological parameters indicative of the refinement process would be highly desirable.

Proton magnetic resonance spectroscopy ( $\left.{ }^{1} \mathrm{H}-\mathrm{MRS}\right)$, a powerful non-invasive technique capable of quantifying biochemical parameters in vivo, has been applied successfully to studies of early cerebral development. These studies have revealed that the levels of N-acetylaspartate (NAA), creatine (Cr), and glutamate (Glu) increase, whereas those of choline (Cho) and myo-inositol decrease during the first few years of life (1-5).
By contrast, several ${ }^{1} \mathrm{H}$-MRS studies of children and adolescents $(1,3,6-9)$ have revealed only subtle age-related changes. These included a slight increase in NAA and a decrease in Cho in several regions $(1,3,6,7)$, the NAA/Cho ratio becoming maximal in the second decade of life $(8,9)$. On the other hand, these previous studies have found no change in the level of Glu. Considering that Glu as an excitatory transmitter is closely associated with the efficacy of synaptic transmission, thereby contributing to refinement of cortical networks (10), Glu may change in association with cortical network refinement during this period.

In the present study, focusing on Glu, we investigated maturational changes in biochemical profiles within the cerebral cortex of normal individuals during childhood and young adulthood using 3.0-T ${ }^{1} \mathrm{H}$-MRS, which allows reliable quantification of Glu owing to its high signal-to-noise ratio and spectral resolution.

\section{METHODS}

\section{Subjects}

Eleven normal volunteers representing the childhood stage (seven male and four female subjects aged $4-13$ years, mean 9.7 years) and eleven normal volunteers representing the young adulthood stage (seven male and four female subjects aged 18-33 years, mean 25.0 years) participated in this study. All procedures were performed in accordance with the human research guidelines of the Internal Review Board of the University of Niigata. Subjects underwent a neurologic examination and interview to confirm the absence of developmental abnormalities and ensure that they were free of any medication and were not taking illicit drugs or alcohol. Written informed consent was obtained from every volunteer or guardian.

\section{Proton MR spectroscopy studies}

A Signa 3.0-T (General Electric, Waukesha, WI) MR system with an eight-element phased array head coil was used to perform all of the studies. $\mathrm{T}_{2}$-weighted fast spin echo images were obtained. PRESS with chemical shift-selective water suppression was utilized with the following parameters: repetition time $=2,000 \mathrm{~ms}$; echo time $=80 \mathrm{~ms}$; data point $=4,096$; spectral width $=5,000 \mathrm{~Hz}$; and 128 signal averages. Using these settings for 3.0-T ${ }^{1} \mathrm{H}-\mathrm{MRS}$, it had been shown previously that resonance at 2.35 parts per million overwhelmingly represents the Glu resonance (11-13). Accordingly, we assigned this resonance to Glu. The overall examination time was $\sim 30 \mathrm{~min}$. During the entire imaging process, volunteers were provided with audiovisual aids and thus adequately prepared. No sedative 


\section{Articles $\mid$ Shimizu et al.}
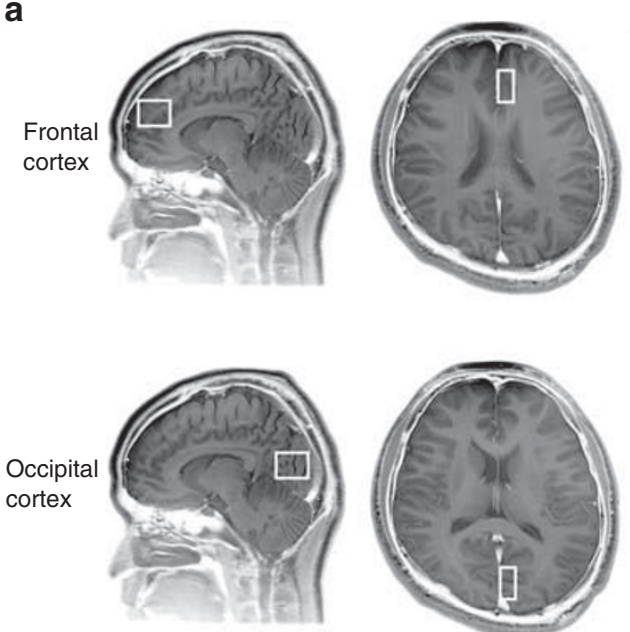

b
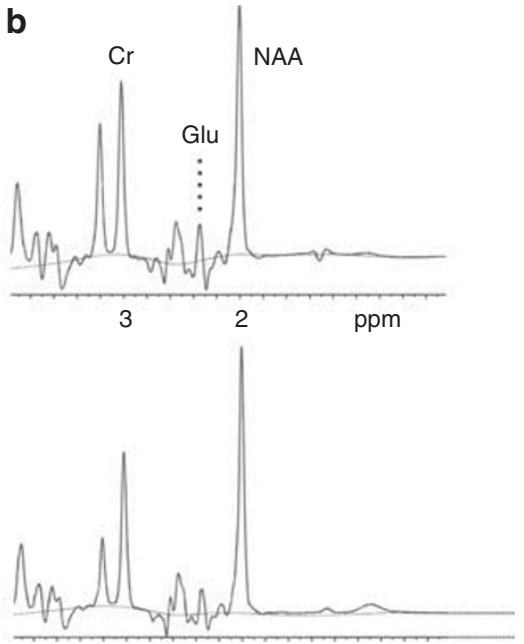

Figure 1. Representative 1H-MRS spectra for each of the ROls. (a) Representative examples of ROls determined from T2-weighted images. ROIs in the frontal and occipital cortices used in this study are demonstrated by white rectangles. (b) Representative $1 \mathrm{H}-\mathrm{MRS}$ spectra with a superimposed Linear Combination Model of in vitro spectra (LCModel) fitted to metabolite concentrations and the estimated baseline. 1H-MRS, proton magnetic resonance spectroscopy; ROI, regions of interest; $\mathrm{Cr}$, creatine; Glu, glutamate; NAA, N-acetylaspartate; ppm, parts per million.
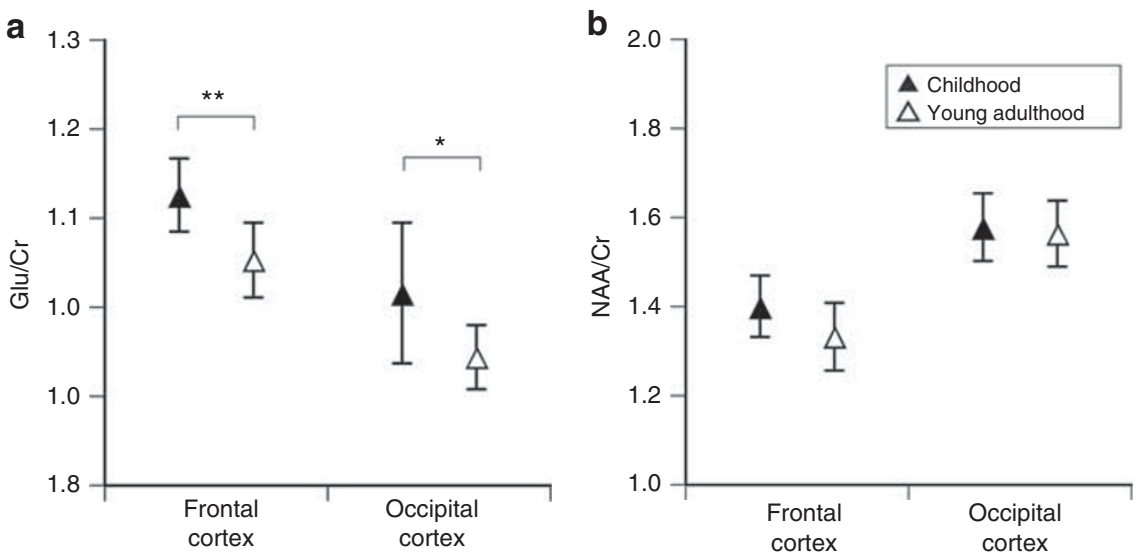

Figure 2. Comparison of $\mathrm{Glu} / \mathrm{Cr}$ and $\mathrm{NAA} / \mathrm{Cr}$ between the two age groups. (a) $\mathrm{Glu} / \mathrm{Cr}$ is significantly smaller at the young adulthood stage than at the childhood stage in the frontal $\left({ }^{*} P<0.01\right)$ and occipital $(* P<0.05)$ cortices. (b) NAA/Cr is not associated with any significant differences between the two age groups. Each symbol and vertical bar represents mean \pm s.d. $\mathrm{Glu} / \mathrm{Cr}$, glutamate to creatine ratio; $\mathrm{NAA} / \mathrm{Cr}$, N-acetylaspartate to creatine ratio.

procedure was performed. ${ }^{1} \mathrm{H}-\mathrm{MRS}$ spectra were obtained from two regions of interest within the frontal and occipital cortices $(20 \times 25 \times 12 \mathrm{~mm}=6 \mathrm{ml}$; Figure 1a).

The Glu/Cr and NAA/Cr ratios were determined by using a Linear Combination Model of in vitro spectra (LCModel, Version 6.2; Stephen Provencher, Oakville, Ontario, Canada; (ref. 14). The criteria used to select the reliable metabolite concentrations were based on the Cramér-Rao lower bounds, which are estimates of the SD of the fit for each metabolite and are also determined by the LCModel. Only results with a Cramér-Rao lower bounds of $<20 \%$ were included in the analyses. Cramér-Rao lower bounds values for metabolites in each brain region were as follows (mean \pm s.d.): in the frontal cortex, Glu 7.1 \pm 1.1 , NAA $2.8 \pm 0.7$, and $\mathrm{Cr} 2.7 \pm 0.5$; in the occipital cortex, Glu $8.8 \pm 1.4$, NAA $3.2 \pm 0.5$, and Cr $3.0 \pm 0.3$.

\section{Statistical analysis}

Comparison of metabolite ratios between the two groups was performed using the Mann - Whitney $U$-test (two-tailed). $P$ values less than 0.05 were considered to represent statistical significance. Analyses were performed using IBM SPSS, version 20.0 (IBM Corporation, Armonk, NY). When significant differences in ratios were found between the two groups, the data were fitted to a logarithmic model, $y=a+b \cdot \ln (x)$, where $y$ is Glu/Cr at age $x$ (in years), and $a$ and $b$ represent the intercept and slope parameters, respectively. Models were determined using SigmaPlot Version 12.5 (SPSS, Chicago, IL).

\section{RESULTS}

Representative ${ }^{1} \mathrm{H}$-MRS spectra are shown in Figure $\mathbf{1 b}$. Quantitative analysis of the Glu-to-creatine ratio $(\mathrm{Glu} / \mathrm{Cr}$ ) and the $\mathrm{N}$-acetylaspartate-to-creatine ratio $(\mathrm{NAA} / \mathrm{Cr})$ is shown in Figure 2. In both of the cerebral cortices, Glu/Cr (mean \pm s.d.) was significantly lower in young adults than in children $(1.05 \pm 0.06$ vs. $1.13 \pm 0.05$ for the frontal cortex, $P<0.01 ; 0.94 \pm 0.05$ vs. $1.02 \pm 0.09$ for the occipital cortex, 


\section{Maturational decrease of brain Glu $\mid$ Articles}
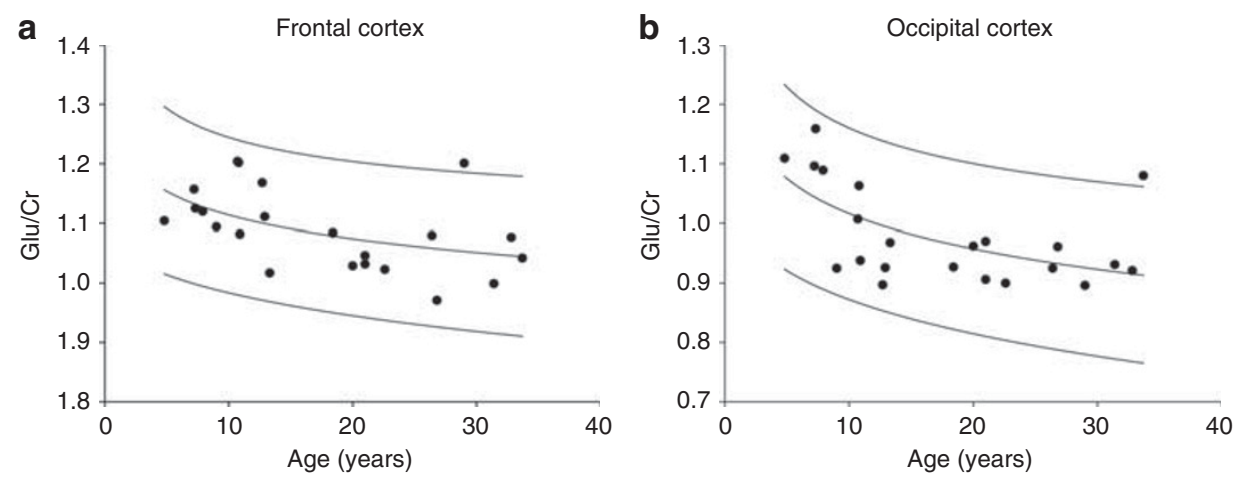

Figure 3. Age-dependent decrease of $\mathrm{Glu} / \mathrm{Cr}$ in the cerebral cortices. (a) Frontal cortex (regression equation: $y=1.25-0.06 \cdot \ln (x)$, all parameters were significant beyond the 0.05 level, $R^{2}=0.23$ ). (b) Occipital cortex (regression equation: $y=1.21-0.08 \cdot \ln (x)$, all parameters were significant beyond the 0.01 level, $R^{2}=0.36$ ). Fitting curves are plotted together with their $95 \%$ prediction intervals. $\mathrm{Glu} / \mathrm{Cr}$, glutamate to creatine ratio; $R^{2}$, $\mathrm{R}$-squared value.

$P<0.05)$. In both cortices, NAA/Cr showed no significant differences between the two age groups. Curve fitting between $\mathrm{Glu} / \mathrm{Cr}$ and age across individual subjects is shown in Figure 3. Glu/Cr decreased with age in both the frontal $(b=-0.06)$ and occipital $(b=-0.08)$ cortices.

\section{DISCUSSION}

The present study has demonstrated that $\mathrm{Glu} / \mathrm{Cr}$ in the cerebral cortex decreases significantly in an age-dependent manner between childhood and young adulthood. Considering that $\mathrm{Cr}$ remains almost constant during this period (3), the significant decline of $\mathrm{Glu} / \mathrm{Cr}$ may reflect a decrease in Glu. A similar maturational Glu decline has been reported in the cerebrum of non-human mammalian species $(15,16)$. Although the precise mechanisms underlying this change remain to be elucidated, it may reflect at least two of the known physiological changes that occur during cerebrum maturation-namely, a decline in synaptic density and a decline in the synthesis of Glu from glutamine, a major precursor of Glu, through alteration of the intercellular compartmentalization of Glu metabolism.

First, during the process of cortical network refinement, unwanted synapses are eliminated. A previous morphometric study has shown that synaptic density in the human cerebral cortex decreases significantly between childhood and young adulthood (17). Considering that the highest Glu concentrations are found inside the presynaptic terminals, a decline in synaptic density may lead to a decrease in Glu. Indeed, in the cornu ammonis of the hippocampus in mice, Glu has been shown to decrease in association with a decrease in synapses (18). Therefore, the decrease in Glu/Cr observed in our study may reflect such a decline in synaptic density.

Second, in the mature brain, metabolism of Glu and its major precursor glutamine is compartmentalized, because several key enzymes and transporters involved in their metabolism are distributed differently in neurons and glia. Consequently, Glu and glutamine are most abundant in neurons and glia, respectively (19). Previous studies of the rat cerebral cortex have demonstrated maturational changes in such enzymes and transporters during synaptic elimination. After the third postnatal week, expression of the glutamine transporter SN1 and activity of glutaminase are significantly decreased $(20,21)$, whereas expression of the Glu transporters (GLAST and GLT-1) and activity of glutamine synthetase appear to be slightly increased (22) and constant (23), respectively, suggesting a maturational decrease in the synthesis of Glu from glutamine, which may account for the decrease in the concentration of Glu. In accordance with these observations, a study of the rat cerebral cortex has demonstrated a decrease in Glu and an increase in glutamine during almost the same period (15). Thus, the decrease in $\mathrm{Glu} / \mathrm{Cr}$ observed in our study may reflect such maturational changes in neuron - glia metabolic interactions with respect to Glu glutamine compartmentalization.

This study has provided objective evidence that cortical $\mathrm{Glu} / \mathrm{Cr}$ decreases between childhood and young adulthood. The observed decrease in $\mathrm{Glu} / \mathrm{Cr}$ may reflect the simultaneous occurrence of maturational changes in the human cerebral cortex. Therefore, evaluation of $\mathrm{Glu} / \mathrm{Cr}$ by in vivo $3.0-\mathrm{T}{ }^{1} \mathrm{H}$ MRS can potentially serve as an indicator of human cerebrum maturation. Further studies employing larger numbers of subjects will be needed to confirm our results, as well as to clarify the effects of sex, sexual maturity, and age on the cerebral Glu concentration. Longitudinal studies or trials using several spectral fitting methods would also be desirable for future practical use.

Human brain maturation is a complex process that involves changes not only in the synapse microstructure but also in axon myelination. Therefore, both gray and white matter should be evaluated to detect and identify regions with abnormal maturation. We propose that Glu/Cr can be used as an indicator of gray matter maturation. We have previously proposed eigenvalue diffusion tensor analysis as an indicator of white matter maturation (24) because it reflects changes in the microstructure, including myelination. We anticipate that use of these in vivo MRI-related indicators will lead to increased understanding of neurodevelopmental and psychiatric disorders. 


\section{Articles | Shimizu et al.}

\section{STATEMENT OF FINANCIAL SUPPORT}

This study was supported by grants from the Ministry of Education, Culture, Sports, Science, and Technology (Japan).

Disclosure: The authors declare no conflict of interest.

\section{REFERENCES}

1. van der Knaap MS, van der Grond J, van Rijen PC, Faber JA, Valk J, Willemse K. Age dependent changes in localized proton and phosphorus MR spectroscopy of the brain. Radiology 1990;176:509-15.

2. Hüppi PS, Posse S, Lazeyras F, Burri R, Bossi E, Herschkowitz N. Magnetic resonance in preterm and term newborns: ${ }^{1} \mathrm{H}$-spectroscopy in developing human brain. Pediatr Res 1991;30:574-8.

3. Kreis R, Ernst T, Ross BD. Development of the human brain: in vivo quantification of metabolite and water content with proton magnetic resonance spectroscopy. Magn Reson Med 1993;30:424-37.

4. Kimura H, Fujii Y, Itoh S, et al. Metabolic alterations in the neonate and infant brain during development: evaluation with proton MR spectroscopy. Radiology 1995;194:483-9.

5. Kreis R, Hofmann L, Kuhlmann B, Boesch C, Bossi E, Hüppi PS. Brain metabolite composition during early human brain development as measured by quantitative in vivo $1 \mathrm{H}$ magnetic resonance spectroscopy. Magn Reson Med 2002;48:949-58.

6. Toft PB, Christiansen P, Pryds O, Lou HC, Henriksen O. T1, T2, and concentrations of brain metabolites in neonates and adolescents estimated with H-1 MR spectroscopy. J Magn Reson Imaging 1994;4:1-5.

7. Pouwels PJ, Brockmann K, Kruse B, et al. Regional age dependence of human brain metabolites from infancy to adulthood as detected by quantitative localized proton MRS. Pediatr Res 1999;46:474-85.

8. Kadota T, Horinouchi T, Kuroda C. Development and aging of the cerebrum: assessment with proton MR spectroscopy. AJNR Am J Neuroradiol 2001;22:128-35.

9. Horská A, Kaufmann WE, Brant LJ, Naidu S, Harris JC, Barker PB. In vivo quantitative proton MRSI study of brain development from childhood to adolescence. J Magn Reson Imaging 2002;15:137-43.

10. Cline HT, Constantine-Paton M. NMDA receptor agonist and antagonists alter retinal ganglion cell arbor structure in the developing frog retinotectal projection. J Neurosci 1990;10:1197-216.

11. Schubert F, Gallinat J, Seifert F, Rinneberg H. Glutamate concentrations in human brain using single voxel proton magnetic resonance spectroscopy at 3 Tesla. Neuroimage 2004;21:1762-71.
12. Ohkubo M, Kimura T, Matsuzawa H, Matsuda T, Kwee IL, Nakada T. Evaluation of efficacy of an automated single-voxel proton MRS algorithm on a 3T system. Magn Reson Med Sci 2002;1: 121-4.

13. Kimura T, Ohkubo M, Igarashi H, Kwee IL, Nakada T. Increase in glutamate as a sensitive indicator of extracellular matrix integrity in peritumoral edema: a 3.0-tesla proton magnetic resonance spectroscopy study. J Neurosurg 2007;106:609-13.

14. Provencher SW. Estimation of metabolite concentrations from localized in vivo proton NMRspectra. Magn Reson Med 1993;30:672-9.

15. Florian CL, Williams SR, Bhakoo KK, Noble MD. Regional and developmental variations in metabolite concentration in the rat brain and eye: a study using 1H NMR spectroscopy and high performance liquid chromatography. Neurochem Res 1996;21:1065-74.

16. Berl S. Compartmentation of glutamic acid metabolism in developing cerebral cortex. J biol chem 1965;240:2047-54.

17. Huttenlocher PR, Dabholkar AS. Regional differences in synaptogenesis in humancerebral cortex. J Comp Neurol 1997;387:167-78.

18. Crescenzi $\mathrm{R}$, DeBrosse $\mathrm{C}$, Nanga $\mathrm{RP}$, et al. In vivo measurement of glutamate loss is associated with synapse loss in a mouse model of tauopathy. Neuroimage 2014;101:185-92.

19. Laake JH, Slyngstad TA, Haug FM, Ottersen OP. Glutamine from glial cells is essential for the maintenance of the nerve terminal pool of glutamate: immunogold evidence from hippocampal slice cultures. J Neurochem 1995;65:871-81.

20. Boulland JL, Rafiki A, Levy LM, Storm-Mathisen J, Chaudhry FA. Highly differential expression of SN1, a bidirectional glutamine transporter, in astroglia and endothelium in the developing rat brain. Glia 2003;41: 260-75.

21. Patel AJ, Hunt A, Hayashi M. Effect of thyroid deficiency on the regional development of glutaminase, a glutamatergic neuron marker, in the rat brain. Int J Dev Neurosci 1987;5:295-303.

22. Furuta A, Rothstein JD, Martin LJ. Glutamate transporter protein subtypes are expressed differentially during rat CNS development. J Neurosci 1997;17:8363-75.

23. Patel AJ, Hunt A, Tahourdin CSM. Regional development of glutamine synthetase activity in the rat brain and its association with the differentiation of astrocytes. Dev Brain Res 1983;8:31-7.

24. Suzuki Y, Matsuzawa H, Kwee IL, Nakada T. Absolute eigenvalue diffusion tensor analysis for human brain maturation. NMR Biomed 2003;16:257-60. 